\title{
Ishikawa iteration process for asymptotic pointwise nonexpansive mappings in metric spaces
}

\section{Buthinah A Bin Dehaish*}

\section{"Correspondence:}

bbindehaish@yahoo.com

Department of Mathematics,

Faculty of Science for Girls, King

Abdulaziz University, Jeddah, 21593,

Saudi Arabia

Department of Mathematical

Sciences, The University of Texas at

El Paso, El Paso, TX 79968, USA

\section{Springer}

\begin{abstract}
Let $(M, d)$ be a complete 2-uniformly convex metric space, $C$ be a nonempty, bounded, closed and convex subset of $M$, and $T$ be an asymptotic pointwise nonexpansive self mapping on $C$. In this paper, we define the modified Ishikawa iteration process in $M$, i.e.,

$$
x_{n+1}=t_{n} T^{n}\left(s_{n} T^{n}\left(x_{n}\right) \oplus\left(1-s_{n}\right)\left(x_{n}\right)\right) \oplus\left(1-t_{n}\right) x_{n}
$$

and we investigate when the Ishikawa iteration process converges weakly to a fixed point of $T$.

MSC: Primary 06F30; 46B20; 47E10

Keywords: asymptotically nonexpansive mapping; asymptotic pointwise nonexpansive mapping; fixed point; inequality of Bruhat and Tits; Ishikawa iteration process; uniformly convex metric space; uniformly Lipschitzian mapping
\end{abstract}

\section{Introduction}

The class of asymptotic nonexpansive mapping have been extensively studied in fixed point theory since the publication of the fundamental paper [1]. Kirk and Xu [2] studied the asymptotic nonexpansive mapping in uniformly convex Banach spaces. Their result has been generalized by Hussain and Khamsi [3] to metric spaces. Khamsi and Kozlowski [4] extended their result to modular function spaces. In almost all papers, authors do not describe any algorithm for constructing a fixed point for the asymptotic nonexpansive mapping. Ishikawa [5] and Mann [6] iterations are two of the most popular methods to check that these two iterations were originally developed to provide ways of computing fixed points for which repeated function iteration failed to converge. Espinola et al. [7] examined the convergence of iterates for asymptotic pointwise contractions in uniformly convex metric spaces. Kozlowski [8] proved convergence to a fixed point of some iterative algorithms applied to asymptotic pointwise mappings in Banach spaces. In [9], the authors discussed the convergence of these iterations in modular function spaces. In a recent paper [10], the authors investigate the existence of a fixed point of asymptotic pointwise nonexpansive mappings and study the convergence of the modified Mann iteration in hyperbolic metric spaces. It is well known that the iteration processes for generalized nonexpansive mappings have been successfully used to develop efficient and powerful numerical meth-

(C) 2013 Bin Dehaish; licensee Springer. This is an Open Access article distributed under the terms of the Creative Commons Attribution License (http://creativecommons.org/licenses/by/2.0), which permits unrestricted use, distribution, and reproduction in any medium, provided the original work is properly cited. 
ods for solving various nonlinear equations and variational problems. The purpose of this paper is to discuss the behavior of the modified Ishikawa iteration process associated with asymptotic pointwise mappings, defined in hyperbolic metric spaces.

For more on metric fixed point theory, the reader may consult the book of Khamsi and Kirk [11].

\section{Basic definitions and results}

Throughout this paper, $(M, d)$ denotes a metric space. Let us assume that there exists a family $\mathcal{F}$ of metric segments such that any two points $x, y$ in $M$ are endpoints of a unique metric segment $[x, y] \in \mathcal{F}([x, y]$ is an isometric image of the real line interval $[0, d(x, y)])$. For any $\beta \in[0,1]$, there exists a unique $z \in[x, y]$ such that

$$
d(x, z)=(1-\beta) d(x, y) \quad \text { and } \quad d(z, y)=\beta d(x, y)
$$

and we will write

$$
z=\beta x \oplus(1-\beta) y .
$$

These metric spaces are usually called convex metric spaces [12]. A hyperbolic metric space is a convex metric space, which satisfies the following condition:

$$
d(\alpha p \oplus(1-\alpha) x, \alpha q \oplus(1-\alpha) y) \leq \alpha d(p, q)+(1-\alpha) d(x, y)
$$

for all $p, q, x, y$ in $M$, and $\alpha \in[0,1]$ (see [13]).

Obviously, the class of hyperbolic spaces includes convex subsets of normed linear spaces. As nonlinear examples, one can consider the CAT(0) spaces [14-16] (see Example 2.1) as well as the Hilbert open unit ball equipped with the hyperbolic metric [17].

Definition 2.1 [17] Let $(M, d)$ be a hyperbolic metric space. We say that $M$ is uniformly convex if for any $a \in M$, for every $r>0$, and for each $\epsilon>0$

$$
\delta(r, \varepsilon)=\inf \left\{1-\frac{1}{r} d\left(\frac{1}{2} x \oplus \frac{1}{2} y, a\right) ; d(x, a) \leq r, d(y, a) \leq r, d(x, y) \geq r \varepsilon\right\}>0 .
$$

Throughout this work, $M$ is a hyperbolic metric space.

The following theorem is a metric version of the parallelogram identity.

Theorem 2.1 [18] Let $(M, d)$ be uniformly convex hyperbolic metric space. Fix a $\in$ M. For each $r>0$ and for each $\varepsilon>0$, denote

$$
\Psi(r, \varepsilon)=\inf \left\{\frac{1}{2} d^{2}(a, x)+\frac{1}{2} d^{2}(a, y)-d^{2}\left(a, \frac{1}{2} x \oplus \frac{1}{2} y\right)\right\},
$$

where the infimum is taken over all $x, y \in M$ such that $d(a, x) \leq r, d(a, y) \leq r$, and $d(x, y) \geq r \varepsilon$. Then $\Psi(r, \varepsilon)>0$ for any $r>0$ and for each $\varepsilon>0$. Moreover, for a fixed $r>0$, we have

(i) $\Psi(r, 0)=0$;

(ii) $\Psi(r, \varepsilon)$ is a nondecreasing function of $\varepsilon$;

(iii) if $\lim _{n \rightarrow \infty} \Psi\left(r, t_{n}\right)=0$, then $\lim _{n \rightarrow \infty} t_{n}=0$. 
Khamsi and Khan [18] used the above function to introduce the nonlinear version of the $p$-uniform convexity in Banach spaces ([19], see also [20], p.310).

Definition 2.2 We say that $(M, d)$ is 2-uniformly convex if

$$
c_{M}=\inf \left\{\frac{\Psi(r, \varepsilon)}{r^{2} \varepsilon^{2}} ; r>0, \varepsilon>0\right\}>0 .
$$

From the definition of $c_{M}$, we obtain the following inequality:

$$
d^{2}\left(a, \frac{1}{2} x \oplus \frac{1}{2} y\right)+c_{M} d^{2}(x, y) \leq \frac{1}{2} d^{2}(a, x)+\frac{1}{2} d^{2}(a, y)
$$

for any $a \in M$ and $x, y \in M$.

Example 2.1 Let $(X, d)$ be a metric space. A geodesic space is a metric space such that every $x, y \in X$ can be joined by a geodesic map $c:[0, l] \rightarrow X$ were $c(0)=x, c(l)=y$, and $d\left(c(t), c\left(t^{\prime}\right)\right)=\left|t-t^{\prime}\right|$ for all $t, t^{\prime} \in[0, l]$. Moreover, $c$ is an isometry and $d(x, y)=l . X$ is said to be uniquely geodesic if for every $x, y \in X$ there is exactly one geodesic joining them, which will be denoted by $[x, y]$, and called the segment joining $x$ to $y$.

A geodesic triangle $\Delta\left(x_{1}, x_{2}, x_{3}\right)$ in a geodesic metric space $(X, d)$ consists of three points $x_{1}, x_{2}, x_{3}$ in $X$ (the vertices of $\Delta$ ) and three geodesic segments between each pair of vertices (the edges of $\Delta$ ). A comparison triangle for $\Delta\left(x_{1}, x_{2}, x_{3}\right)$ in $(X, d)$ is a triangle $\bar{\Delta}\left(x_{1}, x_{2}, x_{3}\right):=$ $\Delta\left(\bar{x}_{1}, \bar{x}_{2}, \bar{x}_{3}\right)$ in $\mathbb{R}^{2}$ such that $d_{\mathbb{R}^{2}}\left(\bar{x}_{i}, \bar{x}_{j}\right)=d\left(x_{i}, x_{j}\right)$ for $i, j \in\{1,2,3\}$. Such a triangle always exists (see [21]).

A geodesic metric space is a $\mathrm{CAT}(0)$ space if every geodesic triangle satisfies the following $\operatorname{CAT}(0)$ inequality:

$$
d(x, y) \leq d(\bar{x}, \bar{y})
$$

for all $x, y \in \Delta$ and all comparison points $\bar{x}, \bar{y} \in \bar{\Delta}$. If $x, y_{1}, y_{2}$ are points of a $\operatorname{CAT}(0)$ space and $y_{0}=\frac{y_{1} \oplus y_{2}}{2}$ is the midpoint of the segment $\left[y_{1}, y_{2}\right]$, then the $\operatorname{CAT}(0)$ inequality implies:

$$
d^{2}\left(x, y_{0}\right) \leq \frac{1}{2} d^{2}\left(x, y_{1}\right)+\frac{1}{2} d^{2}\left(x, y_{2}\right)-\frac{1}{4} d^{2}\left(y_{1}, y_{2}\right)
$$

which is the $(\mathrm{CN})$ inequality of Bruhat and Tits [22]. As for the Hilbert space, the $(\mathrm{CN})$ inequality implies that $\mathrm{CAT}(0)$ spaces are uniformly convex with

$$
\delta(r, \varepsilon)=1-\sqrt{1-\frac{\varepsilon^{2}}{4}} .
$$

One may also find the modulus of uniform convexity via similar triangles. The $(\mathrm{CN})$ inequality also implies that

$$
\Psi(r, \varepsilon)=\frac{r^{2} \varepsilon^{2}}{4} .
$$

This clearly implies that any CAT(0) space is 2-uniformly convex with $c_{M}=\frac{1}{4}$. 
The following inequality plays an important role in the study of the convergence of Ishikawa iterations.

Theorem $2.2[10]$ Assume that $(M, d)$ is 2-uniformly convex. Then for any $\alpha \in(0,1)$, there exists $C_{M}>0$ such that

$$
d^{2}(a, \alpha x \oplus(1-\alpha) y)+C_{M} \min \left(\alpha^{2},(1-\alpha)^{2}\right) d^{2}(x, y) \leq \alpha d^{2}(a, x)+(1-\alpha) d^{2}(a, y),
$$

for any $a, x, y \in M$.

The following technical result is a generalization of Lemma 2.3 of [18].

Lemma 2.1 Let $(M, d)$ be 2-uniformly convex. Assume that $\left\{t_{n}\right\} \subset[0,1]$ be bounded away from 0 and 1, i.e., there exists two real numbers $\alpha, \beta$ such that $0<\alpha \leq t_{n} \leq \beta<1$ and there exists $r \geq 0$ such that

$$
\limsup _{n \rightarrow \infty} d\left(u_{n}, a\right) \leq r, \quad \limsup _{n \rightarrow \infty} d\left(v_{n}, a\right) \leq r \quad \text { and } \quad \lim _{n \rightarrow \infty} d\left(a, t_{n} u_{n} \oplus\left(1-t_{n}\right) v_{n}\right)=r .
$$

Then

$$
\lim _{n \rightarrow \infty} d\left(u_{n}, v_{n}\right)=0 .
$$

Proof By replacing $\alpha$ by $t_{n}, x$ by $u_{n}$ and $y$ by $v_{n}$ in Theorem 2.2, we get

$$
\begin{array}{r}
C_{M} \min \left(t_{n}^{2},\left(1-t_{n}\right)^{2}\right) d^{2}\left(u_{n}, v_{n}\right) \leq \\
t_{n} d^{2}\left(a, u_{n}\right)+\left(1-t_{n}\right) d^{2}\left(a, v_{n}\right) \\
-d^{2}\left(a, t_{n} u_{n} \oplus\left(1-t_{n}\right) v_{n}\right),
\end{array}
$$

for any $a, u_{n}, v_{n} \in M$. Let $\mathcal{U}$ be a nontrivial ultrafilter over $\mathbb{N}$. Then $\lim _{\mathcal{U}} t_{n}=t, t \in[\alpha, \beta]$. Therefore,

$$
\begin{aligned}
C_{M} \min \left(\lim _{\mathcal{U}} t_{n}^{2}, \lim _{\mathcal{U}}\left(1-t_{n}\right)^{2}\right) \lim _{\mathcal{U}} d^{2}\left(u_{n}, v_{n}\right) \leq & \lim _{\mathcal{U}} t_{n} \lim _{\mathcal{U}} d^{2}\left(a, u_{n}\right) \\
& +\lim _{\mathcal{U}}\left(1-t_{n}\right) \lim _{\mathcal{U}} d^{2}\left(a, v_{n}\right) \\
& -\lim _{\mathcal{U}} d^{2}\left(a, t_{n} u_{n} \oplus\left(1-t_{n}\right) v_{n}\right) .
\end{aligned}
$$

Hence,

$$
C_{M} \min \left(t^{2},(1-t)^{2}\right) \lim _{\mathcal{U}} d^{2}\left(u_{n}, v_{n}\right) \leq t r^{2}+(1-t) r^{2}-\lim _{\mathcal{U}}\left(t_{n} d^{2}\left(a, u_{n}\right)+\left(1-t_{n}\right) d^{2}\left(a, v_{n}\right)\right),
$$

which implies

$$
C_{M} \min \left(t^{2},(1-t)^{2}\right) \lim _{\mathcal{U}} d^{2}\left(u_{n}, v_{n}\right)=0 .
$$

But $C_{M}>0, \min \left(t^{2},(1-t)^{2}\right) \neq 0$. Since $t$ is bounded away from 0 and 1 , then

$$
\lim _{\mathcal{U}} d\left(u_{n}, v_{n}\right)=0 .
$$


Since $\mathcal{U}$ was an arbitrary nontrivial ultrafilter over $\mathbb{N}$, we get

$$
\lim _{n \rightarrow \infty} d\left(u_{n}, v_{n}\right)=0 .
$$

Recall that $\tau: M \rightarrow \mathbb{R}_{+}$is called a type if there exists $\left\{x_{n}\right\}$ in $M$ such that

$$
\tau(x)=\limsup _{n \rightarrow \infty} d\left(x, x_{n}\right) .
$$

Theorem 2.3 [18] Assume that $(M, d)$ is complete and uniformly convex. Let $C$ be any nonempty closed, bounded and convex subset of $M$. Let $\tau$ be a type defined on $C$. Then any minimizing sequence of $\tau$ is convergent. Its limit is independent of the minimizing sequence.

In fact, if $M$ is 2-uniformly convex, and $\tau$ is a type defined on a nonempty closed, bounded and convex subset $C$ of $M$, then there exists a unique $x_{0} \in C$ such that

$$
\tau^{2}\left(x_{0}\right)+2 c_{M} d^{2}\left(x_{0}, x\right) \leq \tau^{2}(x),
$$

for any $x \in C$. In this inequality, one may find an analogy with the Opial property used in the study of the fixed point property in Banach and metric spaces.

Definition 2.3 [23] Let $C$ be a nonempty subset of $(M, d)$. A self mapping $T$ on $C$ is said to be asymptotic pointwise nonexpansive if for any $x \in C$, there exists a sequence $\left\{k_{n}(x)\right\} \subset$ $[0, \infty)$ such that

$$
d\left(T^{n}(x), T^{n}(y)\right) \leq k_{n}(x) d(x, y)
$$

for any $y \in C$, and $\lim _{n \rightarrow \infty} k_{n}(x)=1$. A point $c \in C$ is called a fixed point of $T$ if $T(c)=c$. The set of all fixed points of $T$ is denoted by $\operatorname{Fix}(T)$.

Let $x \in C$. Set $K_{n}(x)=\max \left\{k_{n}(x), 1\right\}$. Then we have $K_{n}(x) \geq 1, \lim _{n \rightarrow \infty} K_{n}(x)=1$, and $d\left(T^{n}(x), T^{n}(y)\right) \leq K_{n}(x) d(x, y)$ for all $y$ in $C$, and $n \geq 1$. In other words, in the above definition, we will always assume $K_{n}(x) \geq 1$ for all $n \geq 1$, and $x \in C$.

In [10], the authors gave the following existence fixed point theorem for asymptotic pointwise nonexpansive mappings in hyperbolic metric spaces.

Theorem 2.4 Let $(M, d)$ be a complete hyperbolic metric space which is 2-uniformly convex. Let $C$ be a nonempty, closed, convex and bounded subset of $M$. Let $T$ be an asymptotic pointwise nonexpansive self mapping on $C$. Then $T$ has a fixed point in $C$. Moreover, the fixed point set $\operatorname{Fix}(T)$ is convex.

\section{Ishikawa iteration process}

In this section, we define and prove the weak convergence theorem of the modified Ishikawa iteration process for asymptotic pointwise nonexpansive mappings in a complete hyperbolic 2-uniformly convex metric space $(M, d)$.

Let $C$ be a nonempty, closed, convex and bounded subset of a complete hyperbolic 2-uniformly convex metric space $(M, d)$. Let $T$ be an asymptotic pointwise nonexpansive 
self mapping on $C$. Let $\left\{t_{n}\right\} \subset[0,1]$ be bounded away from 0 and 1 and $\left\{s_{n}\right\} \subset[0,1]$. The modified Ishikawa iteration process is defined by

$$
x_{n+1}=t_{n} T^{n}\left(s_{n} T^{n}\left(x_{n}\right) \oplus\left(1-s_{n}\right)\left(x_{n}\right)\right) \oplus\left(1-t_{n}\right) x_{n},
$$

for any $n \geq 1$, where $x_{1} \in C$ is a fixed arbitrary point, see (cf. [24] and [25]).

In order to prove our main result, the following lemmas are needed.

Lemma 3.1 Let $C$ be a nonempty, closed, convex and bounded subset of a complete hyperbolic 2-uniformly convex metric space $(M, d)$. Let $T$ be an asymptotic pointwise nonexpansive self mapping on $C$. Assume that $\sum_{n=1}^{\infty}\left(k_{n}(x)-1\right)<\infty$, for any $x \in C$, where $\left\{k_{n}(x)\right\}$ is as in Definition 2.3. Let $\left\{t_{n}\right\} \subset[0,1]$ be bounded away from 0 and 1 and $\left\{s_{n}\right\} \subset[0,1]$ in the modified Ishikawa iteration process

$$
x_{n+1}=t_{n} T^{n}\left(s_{n} T^{n}\left(x_{n}\right) \oplus\left(1-s_{n}\right)\left(x_{n}\right)\right) \oplus\left(1-t_{n}\right) x_{n},
$$

where $n \geq 1$ and $x_{1} \in C$ is a fixed arbitrary point. Then for any $\omega \in \operatorname{Fix}(T), \lim _{n \rightarrow \infty} d\left(x_{n}, \omega\right)$ exists.

Proof

$$
\begin{aligned}
d\left(x_{n+1}, \omega\right) & \leq t_{n} d\left(T^{n}\left(s_{n} T^{n}\left(x_{n}\right) \oplus\left(1-s_{n}\right) x_{n}\right), \omega\right)+\left(1-t_{n}\right) d\left(x_{n}, \omega\right) \\
& =t_{n} d\left(T^{n}\left(s_{n} T^{n} x_{n} \oplus\left(1-s_{n}\right) x_{n}\right), T^{n} \omega\right)+\left(1-t_{n}\right) d\left(x_{n}, \omega\right) \\
& \leq t_{n} k_{n}(\omega) d\left(s_{n} T^{n}\left(x_{n}\right) \oplus\left(1-s_{n}\right) x_{n}, \omega\right)+\left(1-t_{n}\right) d\left(x_{n}, \omega\right) \\
& \leq t_{n} k_{n}(\omega)\left[s_{n} d\left(T^{n}\left(x_{n}\right), \omega\right)+\left(1-s_{n}\right) d\left(x_{n}, \omega\right)\right]+\left(1-t_{n}\right) d\left(x_{n}, \omega\right) \\
& \leq t_{n} k_{n}(\omega)\left[s_{n} k_{n}(\omega) d\left(x_{n}, \omega\right)+\left(1-s_{n}\right) d\left(x_{n}, \omega\right)\right]+\left(1-t_{n}\right) d\left(x_{n}, \omega\right) \\
& \leq\left[t_{n} s_{n} k_{n}^{2}(\omega)+\left(1-s_{n}\right) t_{n} k_{n}(\omega)+\left(1-t_{n}\right)\right] d\left(x_{n}, \omega\right) \\
& =\left[t_{n} s_{n} k_{n}(\omega)\left(k_{n}(\omega)-1\right)+t_{n} k_{n}(\omega)+\left(1-t_{n}\right)\right] d\left(x_{n}, \omega\right) \\
& =\left[t_{n} s_{n} k_{n}(\omega)\left(k_{n}(\omega)-1\right)+t_{n}\left(k_{n}(\omega)-1\right)+1\right] d\left(x_{n}, \omega\right) \\
& =\left[t_{n} s_{n} k_{n}(\omega)\left(k_{n}(\omega)-1\right)+t_{n}\left(k_{n}(\omega)-1\right)\right] d\left(x_{n}, \omega\right)+d\left(x_{n}, \omega\right) .
\end{aligned}
$$

Hence,

$$
\begin{aligned}
d\left(x_{n+1}, \omega\right) & \leq\left[k_{n}(\omega)\left(k_{n}(\omega)-1\right)+\left(k_{n}(\omega)-1\right)\right] d\left(x_{n}, \omega\right)+d\left(x_{n}, \omega\right) \\
& =\left(k_{n}(\omega)+1\right)\left(k_{n}(\omega)-1\right) d\left(x_{n}, \omega\right)+d\left(x_{n}, \omega\right) \\
& =\left(k_{n}^{2}(\omega)-1\right) d\left(x_{n}, \omega\right)+d\left(x_{n}, \omega\right) .
\end{aligned}
$$

Now let $\delta(C)=\sup \left\{d\left(c_{1}, c_{2}\right) ; c_{1}, c_{2} \in C\right\}$ be the diameter of $C$. Hence,

$$
d\left(x_{n+m}, \omega\right)-d\left(x_{n}, \omega\right) \leq \delta(C) \sum_{i=0}^{m-1}\left(k_{n+i}^{2}(\omega)-1\right)
$$


for any $n, m \geq 1$. If we let $m \rightarrow \infty$, we get

$$
\limsup _{m \rightarrow \infty} d\left(x_{m}, \omega\right) \leq d\left(x_{n}, \omega\right)+\delta(C) \sum_{i=n}^{\infty}\left(k_{i}^{2}(\omega)-1\right)
$$

for any $n \geq 1$. Note that $\sum_{i=n}^{\infty}\left(k_{i}^{2}(\omega)-1\right)$ is convergent. Indeed since $\lim _{n \rightarrow \infty} k_{n}(\omega)=1$, then $\left(k_{n}(\omega)\right)$ is bounded. Moreover,

$$
k_{n}^{2}(\omega)-1=\left(k_{n}(\omega)-1\right)\left(k_{n}(\omega)+1\right) \leq\left(k_{n}(\omega)-1\right)\left(\sup _{n} k_{n}(\omega)+1\right)
$$

Since $\sum_{i=n}^{\infty}\left(k_{n}(\omega)-1\right)$ is convergent, then $\sum_{i=n}^{\infty} k_{n}^{2}(\omega)-1$ is also convergent.

Next, we let $n \rightarrow \infty$ and get

$$
\begin{aligned}
\limsup _{m \rightarrow \infty} d\left(x_{m}, \omega\right) & \leq \liminf _{n \rightarrow \infty} d\left(x_{n}, \omega\right)+\delta(C) \liminf _{n \rightarrow \infty} \sum_{i=n}^{\infty}\left(k_{i}^{2}(\omega)-1\right) \\
& =\liminf _{n \rightarrow \infty} d\left(x_{n}, \omega\right) .
\end{aligned}
$$

Since $C$ is bounded, we conclude that $\limsup _{m \rightarrow \infty} d\left(x_{m}, \omega\right)=\liminf _{n \rightarrow \infty} d\left(x_{n}, \omega\right)$, which implies the desired conclusion.

Lemma 3.2 Let $(M, d), C$ and $T$ be as in Lemma 3.1. Assume that $\left\{t_{n}\right\} \subset[0,1]$ is bounded away from 0 and 1 , and $\left\{s_{n}\right\} \subset[0,1]$ is bounded away from 1 . Define

$$
y_{n}=s_{n} T^{n}\left(x_{n}\right) \oplus\left(1-s_{n}\right)\left(x_{n}\right),
$$

for any $n \geq 1$. Then

$$
\lim _{n \rightarrow \infty} d\left(x_{n}, T^{n}\left(y_{n}\right)\right)=0
$$

Proof Using Theorem 2.4, $T$ has a fixed point $\omega \in C$. Lemma 3.1 implies that $\lim _{n \rightarrow \infty} d\left(x_{n}\right.$, $\omega)$ exists. Set $r=\lim _{n \rightarrow \infty} d\left(x_{n}, \omega\right)$. Without loss of generality, we may assume $r>0$. Let $\mathcal{U}$ be a nontrivial ultrafilter over $\mathbb{N}$. Then $\lim _{\mathcal{U}} t_{n}=t \in[\alpha, \beta]$ and $\lim _{\mathcal{U}} s_{n}=s \in\left[0, \beta^{*}\right]$, for any $0<\alpha \leq \beta<1$ and $\beta^{*}<1$. Moreover, we have

$$
\begin{aligned}
\lim _{\mathcal{U}} d\left(T^{n}\left(y_{n}\right), \omega\right) & =\lim _{\mathcal{U}} d\left(T^{n}\left(y_{n}\right), T^{n}(\omega)\right) \\
& \leq \lim _{\mathcal{U}} k_{n}(\omega) d\left(y_{n}, \omega\right) \\
& \leq \lim _{\mathcal{U}} k_{n}(\omega) d\left(s_{n} T^{n}\left(x_{n}\right) \oplus\left(1-s_{n}\right)\left(x_{n}\right), \omega\right) \\
& \leq \lim _{\mathcal{U}} k_{n}(\omega)\left[s_{n} d\left(T^{n}\left(x_{n}\right), \omega\right)+\left(1-s_{n}\right) d\left(x_{n}, \omega\right)\right] \\
& \leq \lim _{\mathcal{U}} k_{n}(\omega)\left[s_{n} k_{n}(\omega) d\left(x_{n}, \omega\right)+\left(1-s_{n}\right) d\left(x_{n}, \omega\right)\right] \\
& \leq \lim _{\mathcal{U}}\left[s_{n} k_{n}^{2}(\omega) d\left(x_{n}, \omega\right)+\left(1-s_{n}\right) k_{n}(\omega) d\left(x_{n}, \omega\right)\right] \\
& =\lim _{\mathcal{U}}\left[s_{n} k_{n}^{2}(\omega)+\left(1-s_{n}\right) k_{n}(\omega)\right] d\left(x_{n}, \omega\right) .
\end{aligned}
$$


Therefore,

$$
\lim _{\mathcal{U}} d\left(T^{n}\left(y_{n}\right), \omega\right) \leq(s \cdot 1+(1-s) \cdot 1) r=r .
$$

Since $\mathcal{U}$ was an arbitrary nontrivial ultrafilter over $\mathbb{N}$, we get

$$
\limsup _{n \rightarrow \infty} d\left(T^{n}\left(y_{n}\right), \omega\right) \leq r .
$$

We have

$$
x_{n+1}=t_{n} T^{n}\left(y_{n}\right) \oplus\left(1-t_{n}\right)\left(x_{n}\right),
$$

for any $n \geq 1$, and $\lim _{n \rightarrow \infty} d\left(x_{n+1}, \omega\right)=r$. Using Lemma 2.1, with $u_{n}=T^{n}\left(y_{n}\right)$ and $v_{n}=x_{n}$, we obtain

$$
\lim _{n \rightarrow \infty} d\left(T^{n}\left(y_{n}\right), x_{n}\right)=0 .
$$

Lemma 3.3 Let $(M, d), C, T,\left\{t_{n}\right\}$ and $\left\{s_{n}\right\}$ be as in Lemma 3.2. Then

$$
\lim _{n \rightarrow \infty} d\left(x_{n}, T^{n}\left(x_{n}\right)\right)=0
$$

provided that $L=\sup _{x \in C} k_{n}(x)<\infty$, i.e., $T$ is uniformly Lipschitzian mapping on $C$.

Proof Using Theorem 2.4, $T$ has a fixed point $\omega \in C$. We have from Lemma 3.1

$$
\limsup _{n \rightarrow \infty} d\left(x_{n}, \omega\right)=\lim _{n \rightarrow \infty} d\left(x_{n}, \omega\right)=r .
$$

Moreover, we have

$$
d\left(T^{n}\left(x_{n}\right), \omega\right)=d\left(T^{n}\left(x_{n}\right), T^{n}(\omega)\right) \leq k_{n}(\omega) d\left(x_{n}, \omega\right) .
$$

Hence,

$$
\limsup _{n \rightarrow \infty} d\left(T^{n}\left(x_{n}\right), \omega\right) \leq r .
$$

Let us prove that $\lim _{n \rightarrow \infty} d\left(y_{n}, \omega\right)=r$. Indeed we have

$$
d\left(T^{n}\left(y_{n}\right), \omega\right) \leq k_{n}(\omega) d\left(y_{n}, \omega\right) .
$$

Also

$$
d\left(T^{n}\left(y_{n}\right), \omega\right) \leq d\left(T^{n}\left(y_{n}\right), x_{n}\right)+d\left(x_{n}, \omega\right) .
$$

Using Lemma 3.1 and Lemma 3.2, we get

$$
\limsup _{n \rightarrow \infty} d\left(T^{n}\left(y_{n}\right), \omega\right) \leq r .
$$


Now use (3.1) and (3.5) to get

$$
\begin{aligned}
r & =\lim _{n \rightarrow \infty} d\left(T^{n}\left(y_{n}\right), \omega\right)=\liminf _{n \rightarrow \infty} d\left(T^{n}\left(y_{n}\right), \omega\right) \\
& \leq \liminf _{n \rightarrow \infty} k_{n}(\omega) d\left(y_{n}, \omega\right) \\
& \leq \liminf _{n \rightarrow \infty} d\left(y_{n}, \omega\right) \leq \limsup _{n \rightarrow \infty} d\left(y_{n}, \omega\right) \leq r .
\end{aligned}
$$

Therefore,

$$
\lim _{n \rightarrow \infty} d\left(y_{n}, \omega\right)=\liminf _{n \rightarrow \infty} d\left(y_{n}, \omega\right)=\limsup _{n \rightarrow \infty} d\left(y_{n}, \omega\right)=r
$$

Since $M$ is 2-uniformly convex, (2.2) implies

$$
\begin{aligned}
& d^{2}\left(s_{n} T^{n}\left(x_{n}\right) \oplus\left(1-s_{n}\right) x_{n}, \omega\right)+C_{M} \min \left(s_{n}^{2},\left(1-s_{n}\right)^{2}\right) d^{2}\left(x_{n}, T^{n}\left(x_{n}\right)\right) \\
& \quad \leq s_{n} d^{2}\left(T^{n}\left(x_{n}\right), \omega\right)+\left(1-s_{n}\right) d^{2}\left(x_{n}, \omega\right),
\end{aligned}
$$

which implies

$$
\begin{aligned}
& d^{2}\left(y_{n}, \omega\right)+C_{M} \min \left(s_{n}^{2},\left(1-s_{n}\right)^{2}\right) d^{2}\left(x_{n}, T^{n}\left(x_{n}\right)\right) \\
& \quad \leq s_{n} d^{2}\left(T^{n}\left(x_{n}\right), \omega\right)+\left(1-s_{n}\right) d^{2}\left(x_{n}, \omega\right) .
\end{aligned}
$$

Let $\mathcal{U}$ be a nontrivial ultrafilter over $\mathbb{N}$. Then

$$
\begin{aligned}
& \lim _{\mathcal{U}} d^{2}\left(y_{n}, \omega\right)+C_{M} \lim _{\mathcal{U}} \min \left(s_{n}^{2},\left(1-s_{n}\right)^{2}\right) \lim _{\mathcal{U}} d^{2}\left(x_{n}, T^{n}\left(x_{n}\right)\right) \\
& \quad \leq \lim _{\mathcal{U}} s_{n} d^{2}\left(T^{n}\left(x_{n}\right), \omega\right)+\lim _{\mathcal{U}}\left(1-s_{n}\right) d^{2}\left(x_{n}, \omega\right) .
\end{aligned}
$$

Using Lemma 3.1, relations (3.3) and (3.6), we get

$$
r^{2}+C_{M} \min \left(s^{2},(1-s)^{2}\right) \lim _{\mathcal{U}} d^{2}\left(x_{n}, T^{n}\left(x_{n}\right)\right) \leq s r^{2}+(1-s) r^{2}=r^{2},
$$

where $C_{M}>0$ depends only on $M$. We have

$$
\min \left(s^{2},(1-s)^{2}\right) \lim _{\mathcal{U}} d^{2}\left(x_{n}, T^{n}\left(x_{n}\right)\right) \leq r^{2}-r^{2}=0 .
$$

Therefore,

$$
\min \left(s^{2},(1-s)^{2}\right) \lim _{\mathcal{U}} d^{2}\left(x_{n}, T^{n}\left(x_{n}\right)\right)=0
$$

Now we distinguish two cases for $s$.

Case 1. If $s \neq 0$, then $\lim _{\mathcal{U}} d\left(x_{n}, T^{n}\left(x_{n}\right)\right)=0$.

Case 2. If $s=0$, we have

$$
d\left(y_{n}, x_{n}\right)=d\left(s_{n} T^{n}\left(x_{n}\right) \oplus\left(1-s_{n}\right)\left(x_{n}\right), x_{n}\right)=s_{n} d\left(T^{n}\left(x_{n}\right), x_{n}\right) .
$$


Since $C$ is bounded, we get

$$
\lim _{\mathcal{U}} d\left(y_{n}, x_{n}\right)=s \lim _{\mathcal{U}} d\left(T^{n}\left(x_{n}\right), x_{n}\right)=0
$$

But

$$
d\left(T^{n}\left(y_{n}\right), T^{n}\left(x_{n}\right)\right) \leq L d\left(y_{n}, x_{n}\right)
$$

if used with (3.7), we will get

$$
\lim _{\mathcal{U}} d\left(T^{n}\left(y_{n}\right), T^{n}\left(x_{n}\right)\right) \leq L \lim _{\mathcal{U}} d\left(y_{n}, x_{n}\right)=0
$$

On the other hand, we have

$$
d\left(x_{n}, T^{n}\left(x_{n}\right)\right) \leq d\left(x_{n}, T^{n}\left(y_{n}\right)\right)+d\left(T^{n}\left(y_{n}\right), T^{n}\left(x_{n}\right)\right) .
$$

Hence,

$$
\lim _{\mathcal{U}} d\left(x_{n}, T^{n}\left(x_{n}\right)\right) \leq \lim _{\mathcal{U}} d\left(x_{n}, T^{n}\left(y_{n}\right)\right)+\lim _{\mathcal{U}} d\left(T^{n}\left(y_{n}\right), T^{n}\left(x_{n}\right)\right)
$$

which implies $\lim _{\mathcal{U}} d\left(x_{n}, T^{n}\left(x_{n}\right)\right)=0$. Since $\mathcal{U}$ was an arbitrary nontrivial ultrafilter over $\mathbb{N}$, we get

$$
\lim _{n \rightarrow \infty} d\left(x_{n}, T^{n}\left(x_{n}\right)\right)=0 .
$$

Lemma 3.4 Let $(M, d), C, T,\left\{t_{n}\right\}$ and $\left\{s_{n}\right\}$ be as in Lemma 3.1. Assume $L=\sup _{x \in C} k_{n}(x)<$ $\infty$, i.e., $T$ is uniformly Lipschitzian mapping on $C$. Then

$$
\lim _{n \rightarrow \infty} d\left(x_{n}, T\left(x_{n}\right)\right)=0 .
$$

Proof Note that

$$
d\left(x_{n}, T\left(x_{n}\right)\right) \leq d\left(x_{n}, T^{n}\left(x_{n}\right)\right)+d\left(T^{n}\left(x_{n}\right), T^{n}\left(x_{n}\right)\right),
$$

implies

$$
d\left(x_{n}, T\left(x_{n}\right)\right) \leq d\left(x_{n}, T^{n}\left(x_{n}\right)\right)+L d\left(T^{n-1}\left(x_{n}\right), x_{n}\right),
$$

for any $n \geq 2$. Since

$$
d\left(T^{n-1}\left(x_{n}\right), x_{n}\right) \leq d\left(T^{n-1}\left(x_{n}\right), T^{n-1} x_{n-1}\right)+d\left(T^{n-1}\left(x_{n-1}\right), x_{n}\right),
$$

we get

$$
d\left(T^{n-1}\left(x_{n}\right), x_{n}\right) \leq L d\left(x_{n}, x_{n-1}\right)+d\left(T^{n-1}\left(x_{n-1}\right), x_{n}\right),
$$


for any $n \geq 2$. Moreover, we have

$$
\begin{aligned}
d\left(x_{n}, x_{n-1}\right)= & t_{n-1} d\left(T^{n-1}\left(s_{n-1} T^{n-1}\left(x_{n-1}\right) \oplus\left(1-s_{n-1}\right) x_{n}\right), x_{n-1}\right) \\
& +\left(1-t_{n-1}\right) d\left(x_{n-1}, x_{n-1}\right) \\
= & t_{n-1} d\left(T^{n-1}\left(s_{n-1} T^{n-1}\left(x_{n-1}\right) \oplus\left(1-s_{n-1}\right) x_{n-1}\right), x_{n-1}\right),
\end{aligned}
$$

which implies

$$
d\left(x_{n}, x_{n-1}\right)=t_{n-1} d\left(T^{n-1}\left(y_{n-1}\right), x_{n-1}\right) .
$$

Also, we have

$$
\begin{aligned}
d\left(T^{n-1}\left(x_{n-1}\right), x_{n}\right)= & t_{n-1} d\left(T^{n-1}\left(s_{n-1} T^{n-1}\left(x_{n-1}\right) \oplus\left(1-s_{n-1}\right) x_{n-1}\right), T^{n-1}\left(x_{n-1}\right)\right) \\
& +\left(1-t_{n-1}\right) d\left(x_{n-1}, T^{n-1}\left(x_{n-1}\right)\right) \\
= & t_{n-1} d\left(T^{n-1}\left(y_{n-1}\right), T^{n-1}\left(x_{n-1}\right)\right) \\
& +\left(1-t_{n-1}\right) d\left(x_{n-1}, T^{n-1}\left(x_{n-1}\right)\right),
\end{aligned}
$$

which implies

$$
d\left(T^{n-1}\left(x_{n-1}\right), x_{n}\right) \leq L t_{n-1} d\left(y_{n-1}, x_{n-1}\right)+\left(1-t_{n-1}\right) d\left(x_{n-1}, T^{n-1}\left(x_{n-1}\right)\right) .
$$

Substituting (3.10), (3.11) and (3.12) into (3.9), we get

$$
\begin{aligned}
d\left(x_{n}, T\left(x_{n}\right)\right) \leq & d\left(x_{n}, T^{n}\left(x_{n}\right)\right)+L\left[L t_{n-1} d\left(T^{n-1}\left(y_{n-1}\right), x_{n-1}\right)\right. \\
& \left.+L t_{n-1} d\left(y_{n-1}, x_{n-1}\right)+\left(1-t_{n-1}\right) d\left(x_{n-1}, T^{n-1}\left(x_{n-1}\right)\right)\right] .
\end{aligned}
$$

Let $\mathcal{U}$ be an arbitrary nontrivial ultrafilter over $\mathbb{N}$, then

$$
\begin{aligned}
\lim _{\mathcal{U}} d\left(x_{n}, T\left(x_{n}\right)\right) \leq & \lim _{\mathcal{U}} d\left(x_{n}, T^{n}\left(x_{n}\right)\right)+L\left[L \lim _{\mathcal{U}} t_{n-1} d\left(T^{n-1}\left(y_{n-1}\right), x_{n-1}\right)\right. \\
& \left.+L \lim _{\mathcal{U}} t_{n-1} d\left(y_{n-1}, x_{n-1}\right)+\lim _{\mathcal{U}}\left(1-t_{n-1}\right) d\left(x_{n-1}, T^{n-1}\left(x_{n-1}\right)\right)\right] .
\end{aligned}
$$

Using Lemma 3.2 and (3.7), we get

$$
\lim _{\mathcal{U}} d\left(x_{n}, T\left(x_{n}\right)\right) \leq 0+L[L t \cdot 0+L t \cdot 0+(1-t) \cdot 0]
$$

which implies $\lim _{\mathcal{U}} d\left(x_{n}, T\left(x_{n}\right)\right)=0$, for any nontrivial ultrafilter $\mathcal{U}$ over $\mathbb{N}$. Therefore, we have

$$
\lim _{n \rightarrow \infty} d\left(x_{n}, T\left(x_{n}\right)\right)=0 \text {. }
$$

We conclude this paper by a result connecting the sequence $\left\{x_{n}\right\}$ and $\operatorname{Fix}(T)$. 
Theorem 3.1 Let $(M, d), C, T,\left\{t_{n}\right\}$ and $\left\{s_{n}\right\}$ be as in Lemma 3.1. Define the type $\tau(x)=$ $\limsup _{n \rightarrow \infty} d\left(x_{n}, x\right)$ on $C$. If $\omega$ is the minimum point of $\tau$, i.e., $\tau(\omega)=\inf \{\tau(x) ; x \in C\}$, then $T(\omega)=\omega$.

Proof For any $m, n \geq 1$, we have

$$
d^{2}\left(x_{n}, \frac{\omega \oplus T^{m}(\omega)}{2}\right)+c_{M} d^{2}\left(\omega, T^{m}(\omega)\right) \leq \frac{1}{2} d^{2}\left(x_{n}, \omega\right)+\frac{1}{2} d^{2}\left(x_{n}, T^{m}(\omega)\right) .
$$

If we let $n \rightarrow \infty$, we get

$$
\begin{aligned}
\limsup _{n \rightarrow \infty} d^{2}\left(x_{n}, \frac{\omega \oplus T^{m}(\omega)}{2}\right)+c_{M} d^{2}\left(\omega, T^{m}(\omega)\right) \leq & \frac{1}{2} \limsup _{n \rightarrow \infty} d^{2}\left(x_{n}, \omega\right) \\
& +\frac{1}{2} \limsup _{n \rightarrow \infty} d^{2}\left(x_{n}, T^{m}(\omega)\right) .
\end{aligned}
$$

Using the definition of the type, we get

$$
\tau^{2}\left(\frac{\omega \oplus T^{m}(\omega)}{2}\right)+c_{M} d^{2}\left(\omega, T^{m}(\omega)\right) \leq \frac{1}{2} \tau^{2}(\omega)+\frac{1}{2} \tau^{2}\left(T^{m}(\omega)\right),
$$

for any $m \geq 1$. Since

$$
\tau\left(T^{m}(\omega)\right)=\limsup _{n \rightarrow \infty} d\left(x_{n}, T^{m}(\omega)\right)=\limsup _{n \rightarrow \infty} d\left(T^{m}\left(x_{n}\right), T^{m}(\omega)\right),
$$

we get

$$
\tau\left(T^{m}(\omega)\right) \leq k_{m}(\omega) \limsup _{n \rightarrow \infty} d\left(x_{n}, \omega\right)=k_{m}(\omega) \tau(\omega)
$$

for any $m \geq 1$. Since $\omega$ is the minimum point of $\tau$, we get

$$
\tau(\omega) \leq \tau\left(\frac{\omega \oplus T^{m}(\omega)}{2}\right) .
$$

Hence,

$$
\tau^{2}(\omega)+c_{M} d^{2}\left(\omega, T^{m}(\omega)\right) \leq \frac{1}{2} \tau^{2}(\omega)+\frac{k_{m}^{2}(\omega)}{2} \tau^{2}(\omega),
$$

for any $m \geq 1$. Therefore, we have

$$
c_{M} d^{2}\left(\omega, T^{m}(\omega)\right) \leq \frac{k_{m}^{2}(\omega)-1}{2} \tau^{2}(\omega),
$$

for any $m \geq 1$. This implies that $\lim _{m \rightarrow \infty} d\left(\omega, T^{m}(\omega)\right)=0$. Since

$$
\begin{aligned}
d(\omega, T(\omega)) & \leq d\left(\omega, T^{m+1}(\omega)\right)+d\left(T^{m+1}(\omega), T(\omega)\right) \\
& \leq d\left(\omega, T^{m+1}(\omega)\right)+k_{1}(\omega) d\left(T^{m}(\omega), \omega\right),
\end{aligned}
$$

for any $m \geq 1$, we conclude that $d(\omega, T(\omega))=0$, i.e., $\omega \in \operatorname{Fix}(T)$. 


\section{Competing interests}

The author declares that he has no competing interests.

Received: 19 September 2012 Accepted: 28 March 2013 Published: 12 April 2013

\section{References}

1. Goebel, K, Kirk, WA: A fixed point theorem for asymptotically nonexpansive mappings. Proc. Am. Math. Soc. 35, 171-174 (1972)

2. Kirk, WA, Xu, HK: Asymptotic pointwise contractions. Nonlinear Anal. 69, 4706-4712 (2008)

3. Hussain, N, Khamsi, MA: On asymptotic pointwise contractions in metric spaces. J. Math. Anal. Appl. 71(10), 4423-4429 (2009)

4. Khamsi, MA, Kozlowski, WK: On asymptotic pointwise nonexpansive mappings in modular function spaces. J. Math Anal. Appl. 380(2), 697-708 (2011)

5. Ishikawa, S: Fixed point by a new iteration method. Proc. Am. Math. Soc. 44, 147-150 (1974)

6. Mann, RW: Mean value methods in iteration. Proc. Am. Math. Soc. 4, 506-510 (1953)

7. Espinola, R, Fernandez-Leon, A, Piatek, B: Fixed points of single- and set-valued mappings in uniformly convex metric spaces with no metric convexity. Fixed Point Theory Appl. 2010, Article ID 169837 (2010)

8. Kozlowski, WM: Fixed point iteration processes for asymptotic pointwise nonexpansive mappings in Banach spaces. J. Math. Anal. Appl. 377, 43-52 (2011)

9. Bin Dehaish, BA, Kozlowski, WM: Fixed point iteration process for asymptotic pointwise nonexpansive mapping in modular function spaces. Fixed Point Theory Appl. 2012, 118 (2012)

10. Ibn Dehaish, BA, Khamsi, MA, Khan, AR: Mann iteration process for asymptotic pointwise mapping in metric spaces. J. Math. Anal. Appl. 397, 861-868 (2013)

11. Khamsi, MA, Kirk, WA: An Introduction to Metric Spaces and Fixed Point Theory. Wiley, New York (2001)

12. Menger, K: Untersuchungen über allgemeine Metrik. Math. Ann. 100, 75-163 (1928)

13. Reich, S, Shafrir, I: Nonexpansive iterations in hyperbolic spaces. Nonlinear Anal. 15, 537-558 (1990)

14. Kirk, WA: Fixed point theory for nonexpansive mappings, I and II. In: Fixed Point Theory. Lecture Notes in Mathematics, vol. 886, pp. 485-505. Springer, Berlin (1981)

15. Kirk, WA: A fixed point theorem in CAT(0) spaces and R-trees. Fixed Point Theory Appl. 4, 309-316 (2004)

16. Leustean, L: A quadratic rate of asymptotic regularity for CAT(0)-spaces. J. Math. Anal. Appl. 325, 386-399 (2007)

17. Goebel, K, Reich, S: Uniform Convexity, Hyperbolic Geometry, and Nonexpansive Mappings. Series of Monographs and Textbooks in Pure and Applied Mathematics, vol. 83. Dekker, New York (1984)

18. Khamsi, MA, Khan, AR: Inequalities in metric spaces with applications. Nonlinear Anal. 74, 4036-4045 (2011)

19. Xu, HK: Inequalities in Banach spaces with applications. Nonlinear Anal. 16, 1127-1138 (1991)

20. Beauzamy, B: Introduction to Banach Spaces and Their Geometry. North-Holland, Amsterdam (1985)

21. Bridson, M, Haefliger, A: Metric Spaces of Non-Positive Curvature. Springer, Berlin (1999)

22. Bruhat, F, Tits, J: Groupes réductifs sur un corps local. I. Données radicielles valuées. Publ. Math. IHÉS 41, 5-251 (1972)

23. Kirk, WA: Fixed points of asymptotic contractions. J. Math. Anal. Appl. 277, 645-650 (2003)

24. Khan, AR, Khamsi, MA, Fukhar-ud-din, H: Strong convergence of a general iteration scheme in CAT(0) spaces. Nonlinear Anal. 74, 783-791 (2011)

25. Schu, J: Iterative construction of fixed points of asymptotically nonexpansive mappings. J. Math. Anal. Appl. 158, 407-413 (1991)

doi:10.1186/1687-1812-2013-98

Cite this article as: Bin Dehaish: Ishikawa iteration process for asymptotic pointwise nonexpansive mappings in metric spaces. Fixed Point Theory and Applications 2013 2013:98.

\section{Submit your manuscript to a SpringerOpen ${ }^{\circ}$ journal and benefit from:}

- Convenient online submission

Rigorous peer review

- Immediate publication on acceptance

- Open access: articles freely available online

- High visibility within the field

- Retaining the copyright to your article 